\title{
Fetal development of the human trapezius and sternocleidomastoid muscles
}

\author{
Kwang Ho Cho ${ }^{1, \star}$, Ichiro Morimoto ${ }^{2, \star}$, Masahito Yamamoto ${ }^{2}$, Shinya Hanada ${ }^{2}$, Gen Murakami ${ }^{3}$, \\ Jose Francisco Rodríguez-Vázquez ${ }^{4}$, Shinichi Abe ${ }^{2}$ \\ ${ }^{1}$ Department of Neurology, Wonkwang University School of Medicine and Hospital, Institute of Wonkwang Medical Science, Iksan, Korea, \\ ${ }^{2}$ Department of Anatomy, Tokyo Dental College, Tokyo, ${ }^{3}$ Division of Internal Medicine, Jikou-kai Clinic of Home Visits, Sapporo, Japan, ${ }^{4}$ Department \\ of Anatomy and Human Embryology, Institute of Embryology, Faculty of Medicine, Complutense University, Madrid, Spain
}

\begin{abstract}
At present, there is no photographic evidence of splitting of the trapezius and sternocleidomastoid muscles (SCMs), which share a common anlage that extends caudally toward the limb bud in the embryo at a length of $9 \mathrm{~mm}$. Therefore, the aim of the present study was to identify which structures divide the caudal end of the common anlage at the first sign of splitting into two muscles. In $11 \mathrm{~mm}$-long specimens, the SCM and trapezius muscles were identified as a single mesenchymal condensation. In 15 and $18 \mathrm{~mm}$-long specimens, the SCM and trapezius muscles were separated and extended posteriorly and lymphatic tissues appeared in a primitive lateral cervical space surrounded by the SCM (anterior). In $21 \mathrm{~mm}$-long specimens, the lymphatic vessels were dilated and the accompanying afferents were forming connections with the subcutaneous tissue through a space between the SCM and trapezius muscles. In $27 \mathrm{~mm}$-long specimens, cutaneous lymphatic vessels were evident and had entered the deep tissue between the SCM and trapezius muscles. Vascular dilation may be viewed as a result of less mechanical stress or pressure after muscle splitting.
\end{abstract}

Key words: Fetus, Muscle, Embryology, Lymphatic tissues, Anatomy

Received July 28, 2020; Revised October 11, 2020; Accepted October 29, 2020

\section{Introduction}

The trapezius and sternocleidomastoid muscles (SCMs), which are also referred to as the trapezius-SCM system [1], share a common anlage and are the main muscles innervated by the accessory nerve in the mammalian neck [2]. In mice, the endochondral medial part of the clavicle and part of the sternum originate from the postotic neural crest [3]. Moreover, the myotubes of the trapezius and SCM muscles do not originate from the somite, but rather the lateral plate

\footnotetext{
Corresponding author:

Shinichi Abe (iD)

Department of Anatomy, Tokyo Dental College, Tokyo 101-0061, Japan

E-mail: abesh@tdc.ac.jp
}

*These authors contributed equally to this work. mesoderm [3, 4], in contrast to other skeletal muscles. Splitting of the trapezius and SCM occurs at the caudal part of the common anlage, which extends caudally toward the limb bud when the embryo is about $9 \mathrm{~mm}$ in length [5]. Likewise, a recent study using a three-dimensional reconstruction technique demonstrated splitting of the trapezius and SCM at the caudal end of the common anlage at embryonic day 41 [6]. However, in spite of extensive research, there is no photographic evidence of the splitting of these muscles. Therefore, there is lack of information regarding the topographical anatomy during splitting of the trapezius and SCM muscles. Also, it remains unclear as to whether the developing clavicle and scapula enhance the separation between the trapezius and SCM muscles, and whether caudal extension of the trapezius below the shoulder occurs after splitting. Hence, the aim of the present study was to identify which structures divide the caudal end of the common anlage when splitting of

\section{Copyright (c) 2020. Anatomy \& Cell Biology}

This is an Open Access article distributed under the terms of the Creative Commons Attribution Non-Commercial License (http://creativecommons.org/licenses/by-nc/4.0/) which permits unrestricted non-commercial use, distribution, and reproduction in any medium, provided the original work is properly cited. 
the trapezius and SCM occurs.

\section{Materials and Methods}

The study protocol was approved by the Ethics Committee of the Complutense University of Madrid (approval no. B08/374) and conducted in accordance with the ethical principles described in the Declaration of Helsinki (as revised in Edinburgh 2000). A total of 19 embryos and fetuses at gestational week 6 to 7 (crown-rump length of 15-27 mm) were obtained following miscarriages and ectopic pregnancies managed at the Department of Obstetrics, Complutense University of Madrid (Madrid, Spain). Serial horizontal and sagittal sections of the fetal heads were stained with hematoxylin and eosin, Masson trichrome, or Azan trichrome according to our recent research methods [7-9]. After examination, the stained sections were donated to the collection maintained by the Institute of Embryology, Complutense University of Madrid. During observations, we paid special attention to which structures existed at and around the split- ting site of the SCM and trapezius muscles.

\section{Results}

At Carnegie stage (CS) 16 (6 weeks of development [WD]), the SCM and trapezius muscles were identified as a single mesenchymal condensation (Fig. 1A-C), located at the lateral side of the inferior vagal ganglion (the nodose ganglion) and showed indications of division into the two muscles at the inferior end (Fig. 1D). The accessory nerve which pass lateral to the inferior vagal ganglion entered into a single mesenchymal condensation of the SCM and the trapezius (Fig. 1B, C). Another mesenchymal condensation, a candidate digastricus posterior belly, was located anterior to the upper part of the SCM-trapezius complex (Fig. 1B, C). The posterior cervical muscles (intrinsic back muscles) had not yet divided and were identified together as a single mass.

At CS 18 (7 WD) and CS 20 (8 WD), the SCM and trapezius muscles were separated and extended posteriorly, but connected by a fascia in the upper half. At the inferior half,

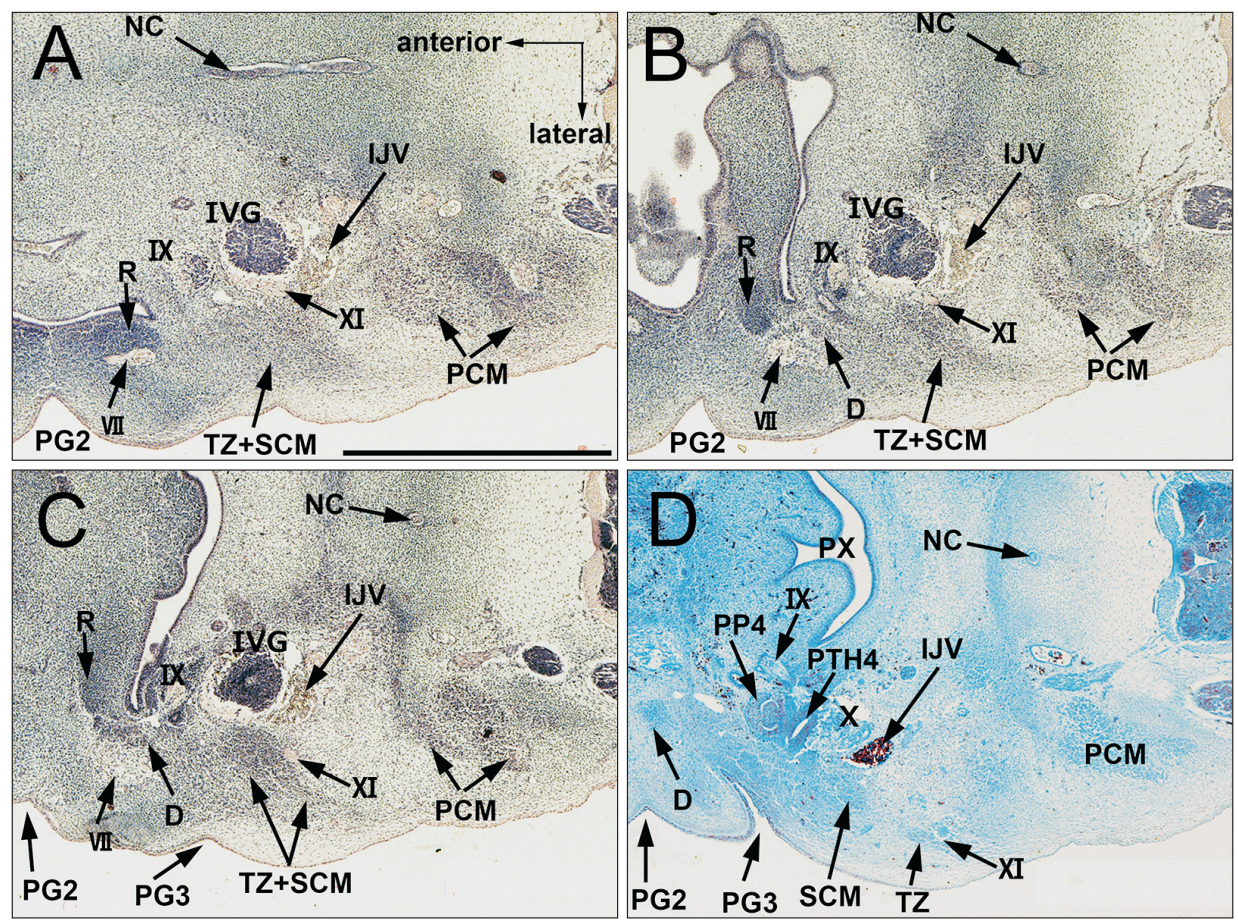

Fig. 1. A single mesenchymal condensation of the SCM and trapezius muscles. CS 16 (6 WD) human embryo (maximum length, 11 mm). Horizontal sections stained with H\&E. (A or D) is the most cranial (or caudal) level of the figure. The SCM and trapezius muscles were identified as a single mesenchymal condensation $(\mathrm{A}-\mathrm{C})$ located at the lateral side of the nodose ganglion, which started dividing into the two muscles at the inferior end (D). CS, Carnegie stage; D, digastric muscle; IJV, internal jugular vein; IVG: inferior vagal ganglion; IX, glossopharyngeal nerve; NC, notochord; PCM, posterior cervical muscles; PG2, second pharyngeal groove; PG3, third pharyngeal groove; PP4, forth pharyngeal pouch; PTH4, parathyroid IV; PX, pharyux; R, Reichert's cartilage (second pharyngeal cartilage); SCM, sternocleidomastoid muscle; TZ, trapezius muscle; VII, facial nerve; XI, accessory nerve; WD, weeks of development. 
the two muscles were identified as the anterior and posterior muscle masses, respectively (Figs. 2B, C, 3B, C). The accessory nerve which ran with the lymphatic vessels innervated the SCM (Fig. 3D). The posterior belly of the digastricus muscle was located between the second pharyngeal cartilage (the Reichert's cartilage) and the SCM (Figs. 2C, D, 3). Although in close proximity, the digastricus muscle was not attached to the SCM (Fig. 3A, B). Lymphatic tissues appeared in a primitive lateral cervical space surrounded by the SCM (anterior), splenius (posterior), internal jugular vein (medial), and trapezius (lateral). The efferent lymphatic vessels ran along the internal jugular vein toward the level of the clavicle (Fig. 2D). In $21 \mathrm{~mm}$-long specimens, the lymphatic vessels were dilated and the associated afferents were forming connections with the subcutaneous tissue through a space between the SCM and trapezius muscles (Fig. 4D). Finally, along the cranial half of the trapezius and SCM in which these muscles were still fused, the splenius increased in thickness and area (Figs. 2B, 3B, 4C).

In $27 \mathrm{~mm}$-long specimens, depending on the upper growth of the digastricus muscle, the splenius muscle was sandwiched between the digastricus and SCM muscles (Fig. 5A, B). The splenius increased so greatly in area that the muscle sheet appeared wavy along the trapezius and SCM. The upper posterior end of the SCM muscle was attached to the splenius muscle, with the latter extending anterolaterally to create a convex-like appearance. The trapezius extended more posteriorly than in the earlier stages of development and provided a large space in front of the SCM muscle (Fig. 5A, B). Cutaneous lymphatic vessels were evident and had entered the deep tissue between the SCM and trapezius muscles (Fig. 5D). Until 7 weeks, the clavicle and scapula occupied almost onethirds of the anteroposterior length of the base of the neck, while the humerus (especially of the head) occupied another one-thirds. During 8 weeks, these cartilage structures reached a length almost same as the left-right length of the base of the neck.

\section{Discussion}

The results of the present study found that splitting of the trapezius and SCM muscles started from the superior part of the common anlage and extended inferiorly. Initial splitting seemed to correspond to the level of the inferior vagal ganglion (the nodose ganglion) of the vagus nerve and the inferior end of the styloid process or the cranial half of Reichert's cartilage. Thus, near the mastoid process, the trapezius and SCM muscles were still combined at 6-7 weeks, while the cartilaginous mastoid portion of the temporal bone was established much later [10]. The SCM and trapezius muscles started to divide in $11 \mathrm{~mm}$-long specimens and the separation was almost complete in 13-15 mm-long specimens (5 weeks). Notably, the posterior belly of the digastricus muscle was located closely to the SCM muscle or the common anlage of the trapezius and SCM muscles. The antero-inferior shift of the digastricus muscle due to formation of the intermediate tendon [11] seemed to drive separation of the SCM and trapezius muscles. Thus, bone formation at the muscle attachment can be affected by the mechanical load from the tendon [12-14]. However, the major driver underlying this
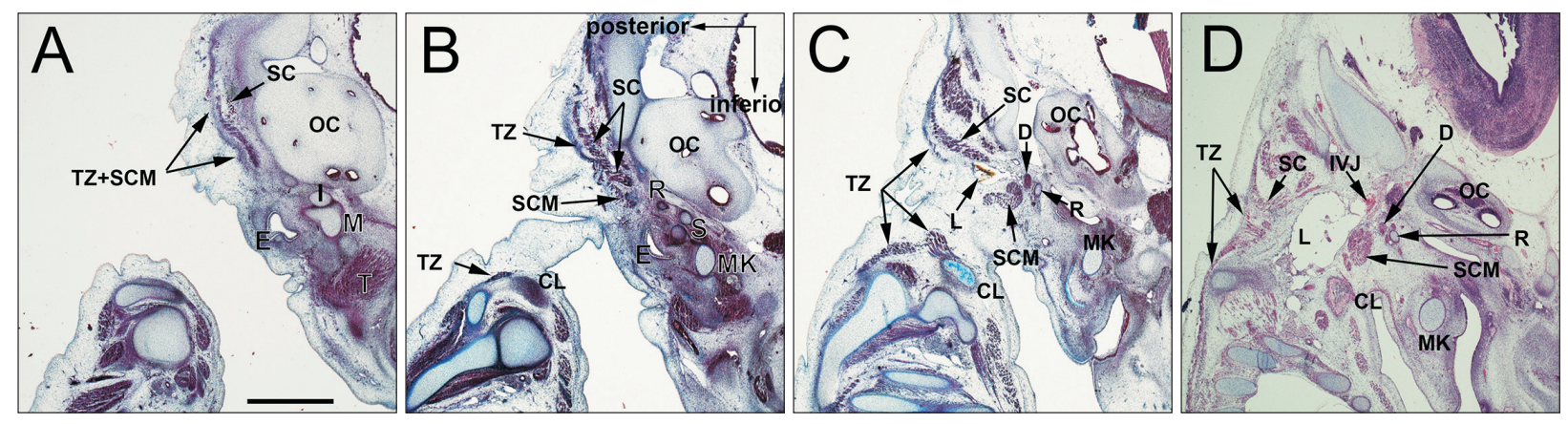

Fig. 2. Differentiation of a single mesenchymal condensation into individual muscles (1). CS 18 (6 WD) human embryo (maximum length, $15 \mathrm{~mm}$ ). Sagittal sections were stained with H\&E. (A or D) is the most lateral (medial) side of the figure. The SCM and trapezius muscles were separated and extended posteriorly, but were connected by a fascia in the upper half (A), while the inferior halves of the two muscles were identified as the anterior and posterior muscle masses, respectively (B, C). The digastricus posterior belly is located between R and the SCM (C, D). CL, clavicle; CS, Carnegie stage; D, digastric muscle; E, external acoustic meatus; I, incus; IJV, internal jugular vein; L, lymphatic vessels; M, mallevs; MK, Meckel's cartilage (first pharyngeal cartilage); OC, Otic capsule; R, Reichert's cartilage (second pharyngeal cartilage); S, stapes; SC, splenius capitis muscle; SCM, sternocleidomastoid muscle; T, temporalis; TZ, trapezius muscle; WD, weeks of development. 

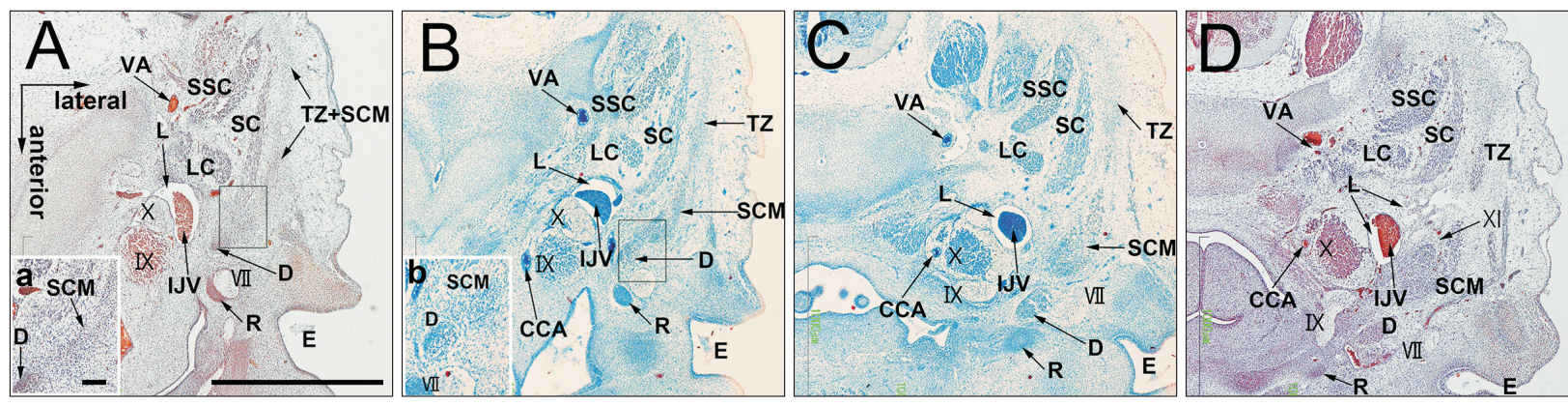

Fig. 3. Differentiation of a single mesenchymal condensation into individual muscles (2). CS 20 (8 WD) human embryo (maximum length, 18 $\mathrm{mm}$ ). Horizontal sections were stained with H\&E. (A or D) is the most cranial (caudal) side of the figure. (a, b) is a high magnification view of the square in (A, B). The SCM and trapezius muscles were separated and extended posteriorly, but connected by a fascia in the upper half. The inferior halves of the two muscles were identified as the anterior and posterior muscle masses, respectively (A-C). The digastricus posterior belly is located between Reichert's cartilage and the SCM (A-D). The digastricus muscle is close, but not attached, to the SCM (a, b). CCA, common carotid artery; CS, Carnegie stage; D, digastric muscle; E, external acoustic meatus; IJV, internal jugular vein; IX, glossopharyngeal nerve; L, lymphatic vessels; LC, longissimus capitis; R, Reichert's cartilage (second pharyngeal cartilage); SC, splenius capitis muscle; SCM, sternocleidomastoid muscle; SSC, semispinalis cervicis; TZ, trapezius muscle; WD, weeks of development; X, vagus nerve; VA, vertebral artery; VII, facial nerve.
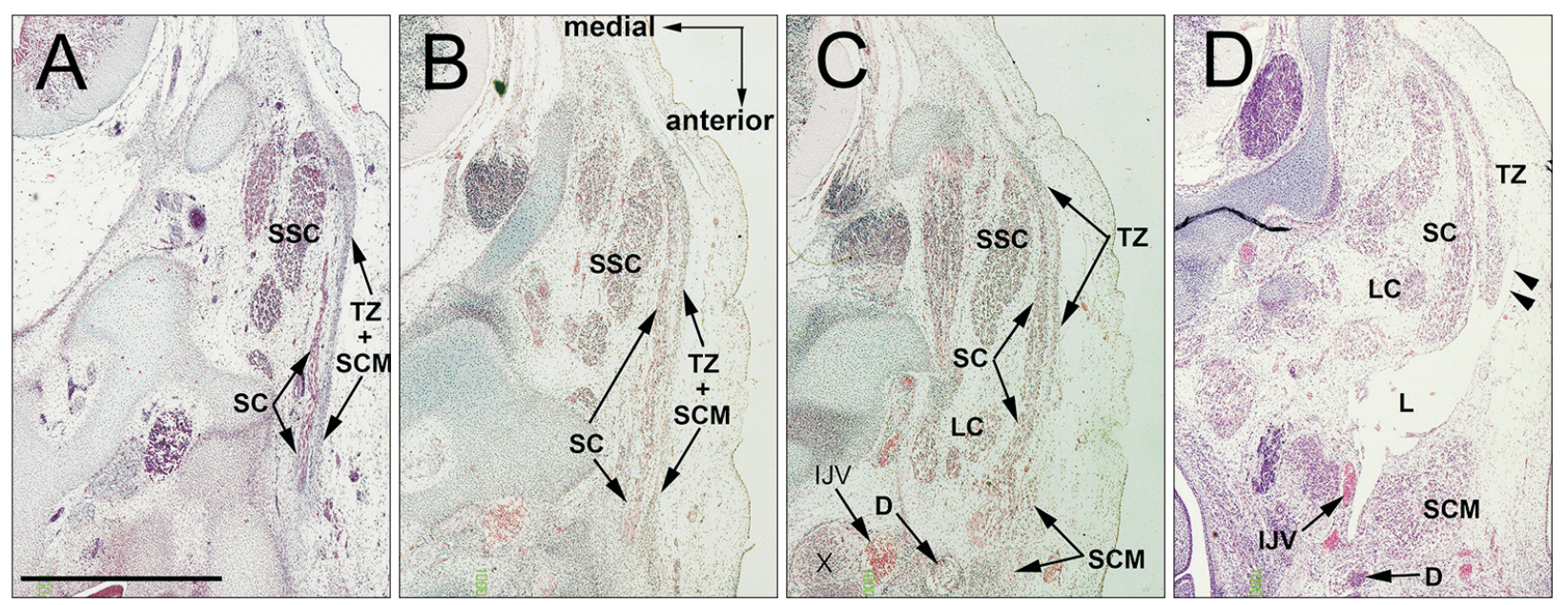

Fig. 4. Lymphatic vessels forming connections with the subcutaneous tissue. CS 20 (8 WD) human embryo (maximum length, 21 mm). Horizontal sections. (A or D) is the most cranial (caudal) side of the figure. The SCM and T extended more posteriorly than in the earlier stages (A-D). The L were dilated and the accompanying afferents formed connections with the subcutaneous tissue through a space between the SCM and TZ (D, arrow heads). CS, Carnegie stage; D, digastric muscle; IJV, internal jugular vein; L, lymphatic vessels; LC, longissimus capitis; SC, splenius capitis muscle; SCM, sternocleidomastoid muscle; SSC, semispinalis cervicis; TZ, trapezius muscle; WD, weeks of development; X, vagus nerve.

separation seemed to be the developing clavicle. Likewise, the developing scapula and the head of the humerus seemed to provide a posterolateral traction to the common anlage or trapezius muscle. In addition, we noted a rapid increase in thickness and area of the splenius along the deep aspect of the trapezius and SCM: the rapid growth might accelerate splitting of the latter two muscles especially at the upper part (Fig. 6).

It is well known that the lesser or smaller occipital nerve sometimes or often penetrates the anterior part of the trapezius muscle. According to Fujita [1], the timing and discrepancy between nerve growth and muscle splitting likely causes the perforating course of the nerve. The course of the lesser occipital nerve acutely changes after perforation [1]. When the nerve passes through the common muscle anlage before or during splitting, the nerve might be pulled more posteriorly than the target area after establishment of the anterior margin of the trapezius muscle. 


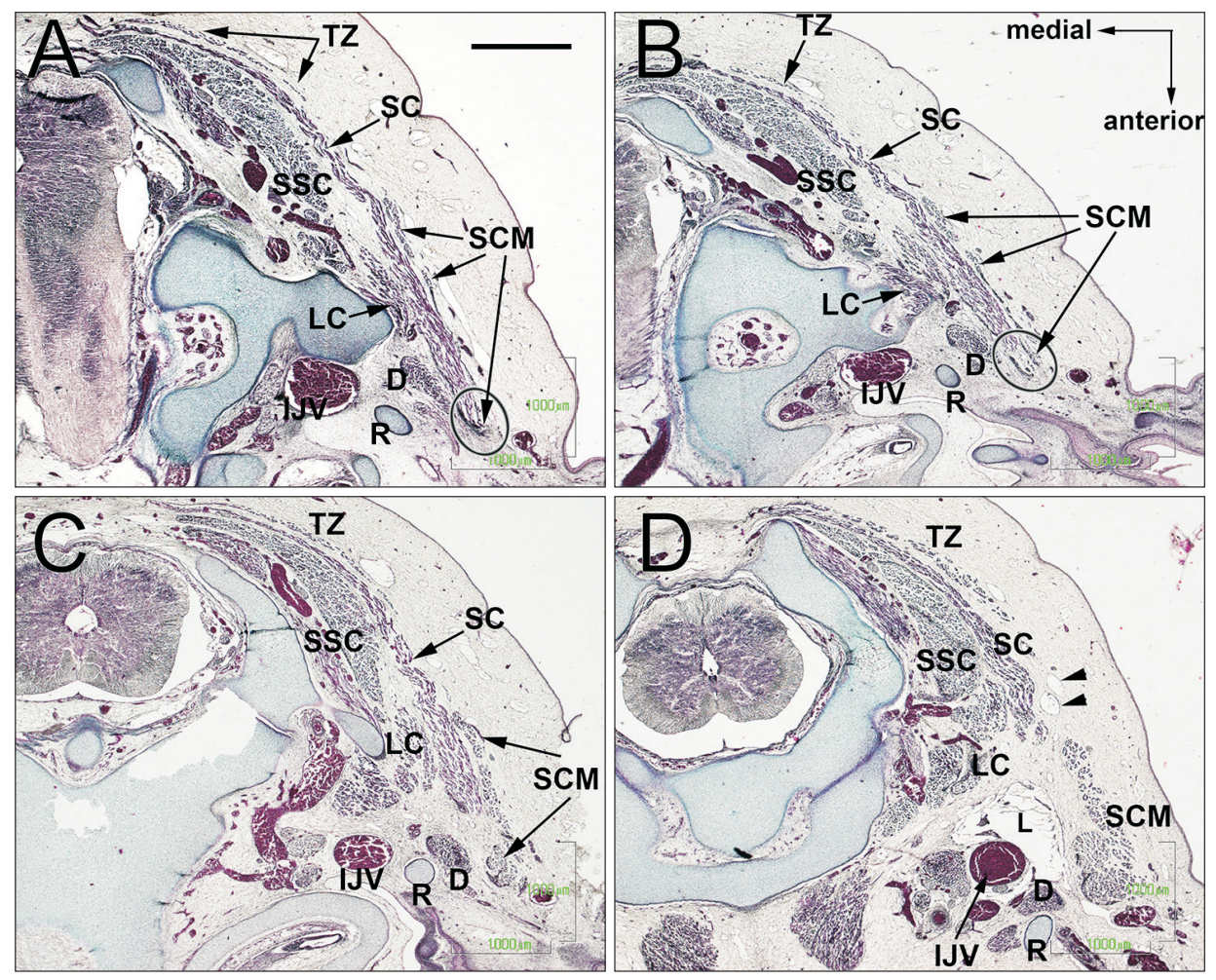

Fig. 5. The upper posterior end of the SCM connecting the splenius capitis muscle. CS 23 (8 WD) human embryo (maximum length, 27 mm). Horizontal sections. (A or D) is the most cranial (caudal) side of the figure. Depending on the upper growth of the digastricus muscle, the splenius muscle was sandwiched between the digastricus and SCM muscles (A, B). The upper posterior end of the SCM was attached to the splenius muscle (A, B, oval). Cutaneous lymphatic vessels had entered the deep tissue between the SCM and trapezius muscles (D, arrow heads). CS, Carnegie stage; D, digastric muscle; IJV, internal jugular vein; LC, longissimus capitis; R, Reichert's cartilage (second pharyngeal cartilage); SC, splenius capitis muscle; SCM, sternocleidomastoid muscle; SSC, semispinalis cervicis; TZ, trapezius muscle; WD, weeks of development.

The embryonic lymphatic vessels were dilated in a loose space between the trapezius and SCM muscles, which may have been a result of less mechanical stress or pressure after splitting of the muscles. Conversely, because the embryonic lymphatic vessels are thin and weak, vascular dilation was unlikely to cause or facilitate splitting of the muscles. At this site, corresponding to the lateral or occipital triangle of the adult neck, the lymphatic vessels are usually well developed even in mid-term fetuses [15]. Moreover, the fetal occipital triangle is a major drainage route from the so-called nuchal translucency (a pathologic or nonpathologic accumulation of great amount of lymphatic fluid in the nuchal subcutaneous tissue) to the venous angle [16]. Pathologically severe nuchal translucency is due to the genetic background and accompanies morphological abnormalities [17]. However, nuchal translucency usually occurs at gestational week 12-16, rather than the embryonic stages, during which the trapezius and SCM are separating [16]. Likewise, the external jugular vein forms a drainage route to the internal jugular or subclavian

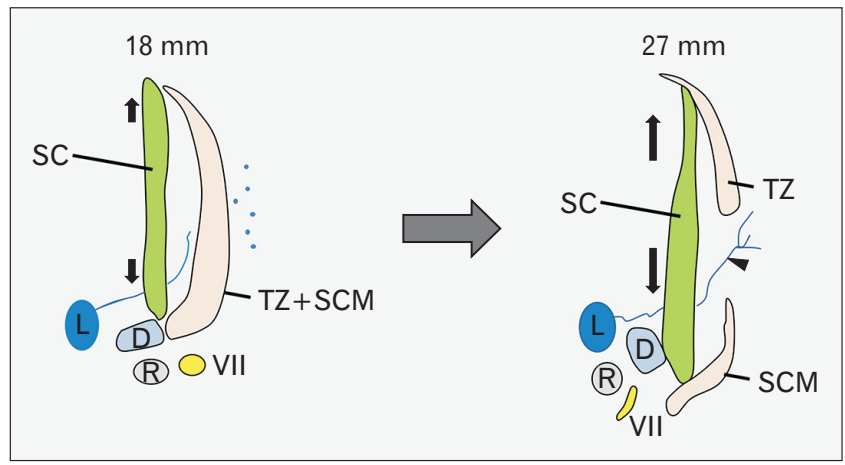

Fig. 6. Schematic representations of the muscle splitting. Growing of the splenius capitis muscle might allow a single mesenchymal condensation of the SCM and trapezius muscles to divide. D, digastric muscle; L, lymphatic vessels; R, Reichert's cartilage (second pharyngeal cartilage); SC, splenius capitis muscle; SCM, sternocleidomastoid muscle; TZ, trapezius muscle; VII, facial nerve.

vein through a loose space between the trapezius and SCM muscles, which also seemed to be a result of splitting.

Consequently, the SCM inserting to the clavicle and the 
trapezius attaching to the scapula were divided soon after development of the upper limb depending on early growth and lateral shift of these cartilage structures. However, the growing digastricus and splenius might accelerate a cranial extension of the muscle splitting (Fig. 6). The splitting allowed a great increase of lymphatic vessels that rescues pathological nuchal lymph edema.

\section{ORCID}

Kwang Ho Cho: https://orcid.org/0000-0002-7751-0469

Ichiro Morimoto: https://orcid.org/0000-0003-4653-836X Masahito Yamamoto:

https://orcid.org/0000-0001-9683-6678

Shinya Hanada: https://orcid.org/0000-0003-0675-4236

Gen Murakami: https://orcid.org/0000-0001-7181-5475

Jose Francisco Rodríguez-Vázquez:

https://orcid.org/0000-0001-5423-4492

Shinichi Abe: https://orcid.org/0000-0003-4632-9736

\section{Author Contributions}

Conceptualization: KHC, IM, GM, JFR, SA. Data acquisition: KHC, IM, GM, JFR, SA. Data analysis or interpretation: KHC, IM, MY, SH, GM. Drafting of the manuscript: KHC, IM, GM. Critical revision of the manuscript: KHC, IM, GM, SA. Approval of the final version of the manuscript: all authors.

\section{Conflicts of Interest}

No potential conflict of interest relevant to this article was reported.

\section{References}

1. Fujita T. The smaller occipital nerve, its topographic relation to the trapezius-sternocleidomastoideus muscle system. Okajimas Folia Anat Jpn 1959;33:217-24.

2. Kuratani S. Evolutionary developmental studies of cyclostomes and the origin of the vertebrate neck. Dev Growth Differ 2008;50(Suppl 1):S189-94.

3. Matsuoka T, Ahlberg PE, Kessaris N, Iannarelli P, Dennehy U, Richardson WD, McMahon AP, Koentges G. Neural crest ori- gins of the neck and shoulder. Nature 2005;436:347-55.

4. Pu Q, Patel K, Huang R. The lateral plate mesoderm: a novel source of skeletal muscle. Results Probl Cell Differ 2015;56:14363.

5. Keibel F, Mall FP. Manual of human embryology. Philadelphia: J.B. Lippincott Company; 1910.

6. Mekonen HK, Hikspoors JP, Mommen G, Eleonore KÖhler S, Lamers WH. Development of the epaxial muscles in the human embryo. Clin Anat 2016;29:1031-45.

7. Yamamoto M, Hashimoto K, Honkura Y, Murakami G, Abe $\mathrm{H}$, Rodríguez-Vázquez JF, Abe SI. Morphology of the upper esophageal sphincter or cricopharyngeus muscle revisited: a study using adult and fetal specimens. Clin Anat 2020;33:78294.

8. Kitamura K, Cho KH, Yamamoto M, Ishii M, Murakami G, Rodríguez-Vázquez JF, Abe SI. Suboccipital myodural bridges revisited: application to cervicogenic headaches. Clin Anat 2019;32:914-28.

9. Sakanaka K, Yamamoto M, Hirouchi H, Kim JH, Murakami G, Rodríguez Vázquez JF, Abe SI. A temporary disc-like structure at the median atlanto-axial joint in human fetuses. Anat Cell Biol 2019;52:436-42.

10. Jin ZW, Cho KH, Abe H, Katori Y, Murakami G, RodríguezVázquez JF. Fetal facial nerve course in the ear region revisited. Surg Radiol Anat 2017;39:885-95.

11. Katori Y, Hyun Kim J, Rodríguez-Vázquez JF, Kawase T, Murakami G, Hwan Cho B. Early fetal development of the intermediate tendon of the human digastricus and omohyoideus muscles: a critical difference in histogenesis. Clin Anat 2011;24:843-52.

12. Nagakura R, Yamamoto M, Jeong J, Hinata N, Katori Y, Chang WJ, Abe S. Switching of Sox9 expression during musculoskeletal system development. Sci Rep 2020;10:8425.

13. Yamamoto M, Abe S. Mechanism of muscle-tendon-bone complex development in the head. Anat Sci Int 2020;95:165-73.

14. Yamamoto M, Takada H, Ishizuka S, Kitamura K, Jeong J, Sato M, Hinata N, Abe S. Morphological association between the muscles and bones in the craniofacial region. PLoS One 2020;15:e0227301.

15. Cho KH, Cheong JS, Ha YS, Cho BH, Murakami G, Katori Y. The anatomy of fetal peripheral lymphatic vessels in the head-and-neck region: an immunohistochemical study. J Anat 2012;220:102-11.

16. Haak MC, van Vugt JM. Echocardiography in early pregnancy: review of literature. J Ultrasound Med 2003;22:271-80.

17. de Mooij YM, van den Akker NM, Bekker MN, Bartelings MM, Wisse LJ, van Vugt JM, Gittenberger-de Groot AC. Abnormal Shh and FOXC2 expression correlates with aberrant lymphatic development in human fetuses with increased nuchal translucency. Prenat Diagn 2009;29:840-6. 